PAPER • OPEN ACCESS

\title{
Eigenmodes of a disordered FeCo magnonic crystal at finite temperatures
}

To cite this article: S Paischer et al 2021 J. Phys.: Condens. Matter 33335804

View the article online for updates and enhancements.

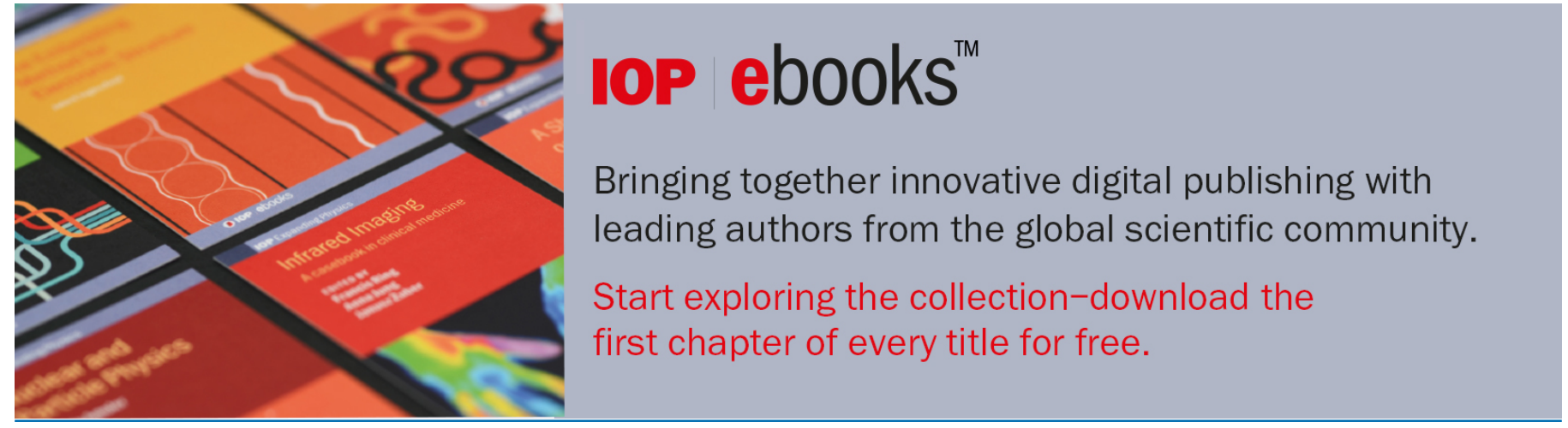

This content was downloaded from IP address 140.78 .241 .158 on 16/07/2021 at 10:32 


\title{
Eigenmodes of a disordered FeCo magnonic crystal at finite temperatures
}

\author{
S Paischer ${ }^{1, *}$ (1), P A Buczek², N Buczek ${ }^{3}$, D Eilmsteiner ${ }^{1,2}$ and A Ernst ${ }^{1,4}$ \\ ${ }^{1}$ Institute for Theoretical Physics, Johannes Kepler University Linz, Altenberger Straße 69, 4040 Linz, \\ Austria \\ ${ }^{2}$ Department of Engineering and Computer Sciences, Hamburg University of Applied Sciences, Berliner \\ Tor 7, 20099 Hamburg, Germany \\ 3 Department of Applied Natural Sciences, Lübeck University of Applied Sciences, Mönkhofer Weg 239, \\ 23562 Lübeck, Germany \\ ${ }^{4}$ Max-Planck-Institut of Microstructure Physics, Weinberg 2, 06120 Halle (Saale), Germany \\ E-mail: sebastian.paischer@jku.at
}

Received 12 March 2021, revised 19 May 2021

Accepted for publication 8 June 2021

Published 25 June 2021

\begin{abstract}
In this report we present a systematic study of the magnonic modes in the disordered $\mathrm{Fe}_{0.5} \mathrm{Co}_{0.5}$ alloy based on the Heisenberg Hamiltonian using two complementary approaches. In order to account for substitutional disorder, on the one hand we directly average the transverse magnetic susceptibility in real space over different disorder configurations and on the other hand we use the coherent potential approximation (CPA). While the method of direct averaging is numerically exact, it is computationally expensive and limited by the maximal size of the supercell which can be simulated on a computer. On the contrary the CPA does not suffer from this drawback and yields a cheap numerical scheme. Therefore, we additionally compare the results of these two approaches and show that the CPA gives very good results for most of the magnetic properties considered in this report, including the magnon energies and the spatial shape of the eigenmodes. However, it turns out that while reproducing the general trend, the CPA systematically underestimates the disorder induced damping of the magnons. This provides evidence that the physics of impurity scattering in this system is governed by non-local effects missing in the CPA. Finally, we study the real space eigenmodes of the system, including their spatial shapes, and analyze their temperature dependence within the random phase approximation.
\end{abstract}

Keywords: alloy, eigenmodes, FeCo, magnon, disorder

(Some figures may appear in colour only in the online journal)

\section{Introduction}

Over the last few decades, the field of magnon spintronics, or magnonics, gained an ever increasing amount of attention. This novel strategy of data propagation and processing has several advantages over the commonly used electronic circuits, like a lack of energy loss through Joule heating [1].

* Author to whom any correspondence should be addressed.

(c) (i) Original content from this work may be used under the terms of the Creative Commons Attribution 4.0 licence. Any further distribution of this work must maintain attribution to the author(s) and the title of the work, journal citation and DOI.
The elemental information carriers are spin waves (also called magnons) which can be pictured as a coherent precession of the magnetic moments in the material [2]. Similar to phonons, magnons are Bloch waves in periodic systems carrying a crystal momentum and energy. However, in order to construct a magnonic circuit, one depends on suitable materials, referred to as magnonic crystals $[3,4]$. The most common magnetic materials are not suited for the use in magnonics since they lack desired properties, especially the emergence of a magnonic bandgap (i.e. frequency bands in which magnon states cannot propagate in the solid $[5,6])$. Combined with the unique spin wave dispersion close to the band edges, this feature provides 
a rich toolbox for magnon mode engineering, including the possibility of selective spin wave excitations and propagation, magnon mode confinement and deceleration, and bandgap soliton generation [7-9].

In current research, mostly long wavelength magnons with energies in the gigahertz band are studied. However, in principle magnons in the terahertz regime are preferred for magnonic applications as they warrant faster information transport and smaller devices [10]. In materials with many magnetic atoms in the primitive unit cell, one expects the occurrence of several magnon modes, which might be separated by a bandgap, yielding a natural magnonic crystal [11].

Here, we concentrate on the ferromagnetic $\mathrm{Fe}_{0.5} \mathrm{Co}_{0.5}$ alloy. This alloy shows all the necessary properties for a terahertz magnonic crystal: its typical magnon energies are well within the terahertz range, it has a high Curie temperature [12, 13], and coherent potential approximation (CPA) studies suggest the spectrum to exhibit a bandgap which remains stable at elevated temperatures [14]. It is interesting to note that magnonic crystals used in gigahertz applications are typically artificial heterostructures obtained from elaborate fabrication processes [3]. On the contrary, in the terahertz range, the natural microscopic arrangement of atoms in alloys like $\mathrm{Fe}_{x} \mathrm{Co}_{1-x}$ would suffice to create cheap magnonic crystals.

In real materials, there are several mechanisms that influence the lifetimes of magnons. First, the interaction of magnons with electronic excitations including a spin flip, called Stoner excitations, plays an essential role especially in metals [15-17]. This mechanism, called Landau damping, was shown to be affected by reduced dimensionality of the system and alloying [18].

Second, the scattering on crystal imperfections might influence the magnon lifetimes as well. In our recent report [14] we showed that this effect may lead to non-trivial dependence of the magnon damping in iron cobalt alloys when the concentration of cobalt is varied. Finally, a non zero temperature is expected to reduce the lifetimes of magnons in the system. Materials to be used for magnonic devices have to operate well above room temperature and feature structural imperfections, as every solid does. Thus, it is interesting to investigate how the magnonic properties evolve in real, imperfect or alloyed solids at non-zero temperatures. We analyze the alloy $\mathrm{Fe}_{0.5} \mathrm{Co}_{0.5}$ using two different approaches: on the one hand, we directly average the transverse magnetic susceptibility over several disordered configurations. Only substitutional disorder is considered which is generated using pseudorandom numbers. Therefore we resort to this method as Monte Carlo (MC) method in the following. On the other hand we utilize a CPA applied to the disordered Heisenberg ferromagnet [19]. This mean field approach was successfully applied for the calculation of electronic and magnetic properties in numerous materials, e.g. [19-21]. The superiority of our CPA method compared to other treatments of the same problem is the possibility to account for complex crystal structures. To incorporate finite temperature effects, we implemented a modified version of the random phase approximation (RPA) discussed in reference [22]. While the RPA method accounts quantitatively for the softening of the magnon modes with temperature, it does not describe the reduction of the magnon life-time due to the interaction of these modes with the thermal bath mentioned above. We show that both MC and CPA methods give the same magnetic spectrum and the spatial shape of the eigenmodes. The only discrepancy between the two methods appears in the magnitude of disorder-induced damping which is clearly underestimated within the CPA. Furthermore, we analyze the real space representation of the dominant eigenmode at $200 \mathrm{meV}$ and show that, with the RPA, the spatial shape of the modes are basically unaltered by increasing temperatures. Our formalism does not include the Landau damping of the spin waves. This attenuation mechanism can be pronounced in metallic magnonic crystals and can be described within the framework of many-body perturbation theory [23] or timedependent density functional theory $[16,24,25]$. Although the dynamical magnetic susceptibility can be calculated for disordered materials within a CPA method [24, 25], the approach requires careful numerical analysis and is subject of a separate study.

The paper is organized as follows: in chapter 2, the theoretical background of the RPA-CPA theory and the MC method for the disordered Heisenberg ferromagnet are discussed. The obtained results are presented in chapter 3 .

\section{Theory}

We deploy the following form of the Heisenberg Hamiltonian

$$
H=-\frac{1}{2} \sum_{i, j} J_{i j} \boldsymbol{e}_{i} \cdot \boldsymbol{e}_{j},
$$

where $J_{i j}$ are the exchange parameters which were obtained from the magnetic force theorem [26] and $\boldsymbol{e}_{i}$ is a unit vector in the direction of the magnetization at site $i$. Anisotropy terms are neglected on the energy scales relevant for this study. To calculate magnon properties, the transverse magnetic susceptibility [27]

$$
\chi_{i j}\left(t, t^{\prime}\right)=-i \Theta\left(t-t^{\prime}\right) \overline{\left[\mu_{i}^{+}(t), \mu_{j}^{-}\left(t^{\prime}\right)\right]}
$$

with $\mu_{i}^{ \pm}=\mu_{i}^{x} \pm i \mu_{i}^{y}, \mu_{i}^{\alpha}$ being the $\alpha$-component of the magnetic moment $\boldsymbol{\mu}_{i}$ on the lattice site $i$ and the overline represents a thermal average, is computed. It can be found by solving the equation of motion

$$
\begin{aligned}
z \chi_{i j}(z)= & 2 g \delta_{i j} \bar{\mu}_{i}-g \sum_{\ell} \frac{\bar{\mu}_{i}}{\mu_{i} \mu_{\ell}} J_{i \ell} \chi_{\ell j}(z) \\
& +g \sum_{\ell} \frac{\bar{\mu}_{\ell}}{\mu_{i} \mu_{\ell}} J_{i \ell} \chi_{i j}(z)
\end{aligned}
$$

with the energy $z=E+i \epsilon$ and the Landé factor $g$. In the following, we assume a material with a complex structure and use an argument $\boldsymbol{R}$ to specify the primitive unit cell, a latin index to specify the basis site and a greek index to distinguish between different atomic species. The disorder is modeled by defining 
occupation variables

$$
p^{i \alpha}(\boldsymbol{R})= \begin{cases}1 & \text { species } \alpha \text { on basis site } i \text { in unit cell } \boldsymbol{R} \\ 0 & \text { else }\end{cases}
$$

and a species resolved Fourier transformation

$$
\begin{aligned}
\chi_{i j}^{\alpha \beta}\left(z, \boldsymbol{k}, \boldsymbol{k}^{\prime}\right):= & \sum_{\boldsymbol{R}, \boldsymbol{R}^{\prime}} p^{i \alpha}(\boldsymbol{R}) \mathrm{e}^{-\mathrm{i} \boldsymbol{k} \cdot \boldsymbol{R}} \\
& \times \chi_{i j}\left(\boldsymbol{R}, \boldsymbol{R}^{\prime}\right) p^{j \beta}\left(\boldsymbol{R}^{\prime}\right) \mathrm{e}^{\mathrm{i} \boldsymbol{k}^{\prime} \cdot \boldsymbol{R}^{\prime}} .
\end{aligned}
$$

We calculate this species resolved susceptibility and average it using the MC and CPA methods described in more detail later in this section.

The averaged susceptibility $\mathcal{X}$ is used to calculate the loss matrix

$$
\mathcal{L}(z, \boldsymbol{k})=\frac{1}{2 i}\left(\mathcal{X}(z, \boldsymbol{k})-\mathcal{X}^{\dagger}(z, \boldsymbol{k})\right),
$$

which holds the information about the elementary excitations of the system. Its eigenvalues are non zero at the energies and wavevectors at which elementary excitations, i.e. magnons, occur. The eigenvectors at the corresponding energy and wavevector yield the shape of the excitation in question. The components of the eigenvectors are to be interpreted as the transverse component of the magnetic moments induced during the moment's precession (magnon excitation). The fluctuation-dissipation theorem [28] states that the excited states of the system are intrinsically related to the linear response of the system upon application of the corresponding external perturbation. Therefore, the shape of magnonic modes can be inferred from the analysis of the response of the magnet to an external magnetic field, in this case in the direction perpendicular to the ground state magnetization.

In the linear regime, the small angle $\theta$ between the magnetic moments and the $z$ axis (giving the direction of the ground state magnetization) depends on the strength of the external perturbation, cf figure 1. It is zero in the ground state. Suppose the projection of the tilted moments to the $x y$ plane be $\epsilon \tilde{\mu}_{i \alpha}$ with a small parameter $\epsilon$ linearly dependent on the strength of the external field. The component of the (normalized) eigenvector of the loss matrix is $\tilde{\mu}_{i \alpha}$. Then

$$
\theta_{i \alpha} \approx \sin \left(\theta_{i \alpha}\right)=\frac{\epsilon \tilde{\mu}_{i \alpha}^{x y}}{\mu_{i \alpha}} .
$$

The ratio between the angles $\theta_{i \alpha}$ of different constituents is independent of $\epsilon$.

$$
\frac{\theta_{i \alpha}}{\theta_{j \beta}}=\frac{\tilde{\mu}_{i \alpha} \mu_{j \beta}}{\tilde{\mu}_{j \beta} \mu_{i \alpha}} .
$$

We model the temperature dependence by a generalized version of the RPA introduced by Callen [22] for simple ordered systems. The thermally averaged magnetic moments are evaluated by calculating

$$
\bar{\mu}=g \frac{\left(\frac{\mu}{g}-\Phi\right)(1+\Phi)^{\mu+1}+\left(\frac{\mu}{g}+1+\Phi\right) \Phi^{\mu+1}}{(1+\Phi)^{\mu+1}-\Phi^{\mu+1}}
$$

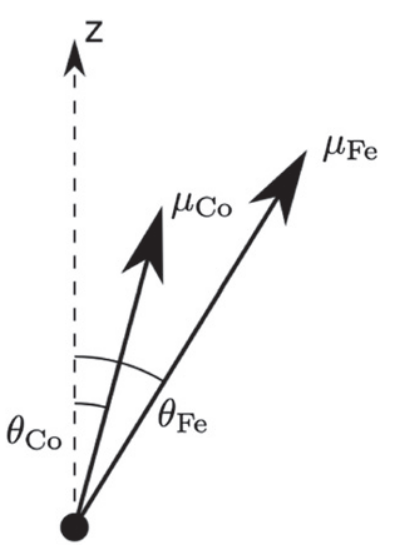

Figure 1. Angles in the case of the iron cobalt alloy.

once for every species for CPA, and once for every site in the system for MC calculations. Here

$$
\Phi=\int_{-\infty}^{\infty}-z \frac{D(z)}{\mathrm{e}^{\frac{z}{k_{\mathrm{B}} T}}-1}
$$

with the density of states $D(z)$ for the species or lattice site in question.

\subsection{MC calculations}

For the direct numerical averaging of the susceptibility, we use a supercell with $20 \times 20 \times 20$ primitive unit cells and periodic boundary conditions. The generation of the random occupation is done by means of pseudorandom numbers. The obtained real space susceptibility is averaged over 30 different configurations. With these parameters, the results are converged. They will be presented in chapter 3 . The susceptibility of each configuration is given in real space by

$$
\chi=M^{-1} \mu,
$$

where bold symbols denote matrices in the site basis and

$$
\begin{aligned}
\mu_{i j} & =2 g \delta_{i j} \bar{\mu}_{i} \\
M_{i j} & =z \delta_{i j}+g \frac{\bar{\mu}_{i}}{\mu_{i} \mu_{j}} J_{i j}-g \sum_{m} \frac{\bar{\mu}_{m}}{\mu_{i} \mu_{m}} J_{i m} \delta_{i j} .
\end{aligned}
$$

\subsection{Coherent potential approximation}

Within the CPA formalism, the equation of motion 3 is used to generate a series expansion of the susceptibility. The series is the Fourier transformed using equation (5), which leads to the series written diagrammatically in figure 2(a)) [29]. The following symbols are used:

- The $\tau$-matrix

$$
\begin{aligned}
\tau_{(i)(j)}^{(\ell)}\left(\boldsymbol{k}, \boldsymbol{k}^{\prime}\right)= & g \mu_{(j)}^{-1}\left(J_{(j)(\ell)}\left(\boldsymbol{k}-\boldsymbol{k}^{\prime}\right) \frac{\bar{\mu}_{(\ell)}}{\mu_{(\ell)}} \delta_{(i)(j)}\right. \\
& \left.-J_{(\ell)(j)}\left(\boldsymbol{k}^{\prime}\right) \frac{\bar{\mu}_{(i)}}{\mu_{(i)}} \delta_{(i)(\ell)}\right)
\end{aligned}
$$


a) $\quad \chi_{(i)(j)}=\longrightarrow \mathrm{C}+\longrightarrow \mathrm{\longrightarrow}+\longrightarrow \mathrm{C}+\cdots$

b)

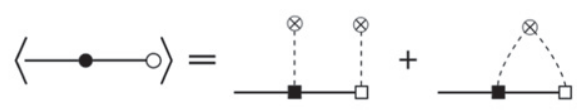

c)

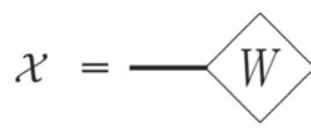

d)

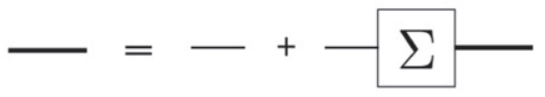

e)

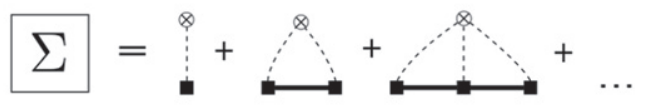

f)

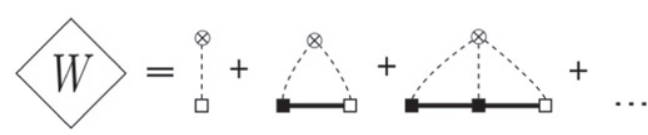

Figure 2. Diagrammatic representation of the main results of the CPA-theory. (a) Fourier transformation of series (3), (b) average of the second term in (a), (c) the averaged susceptibility $\mathcal{X}$ written as a product of the effective medium propagator $\Xi$ (thick line) and the spin weight $W$, (d) Dyson equation for the effective medium propagator, (e) definition of the self-energy $\Sigma$ and (f) definition of the spin weight $W$.

where

$$
J_{(i)(j)}(\boldsymbol{k})=\sum_{\boldsymbol{R}} J_{(i)(j)}(\boldsymbol{R}) \mathrm{e}^{-\mathrm{i} \boldsymbol{k} \cdot \boldsymbol{R}}
$$

is represented by a filled square.

- The filled circle represents a $T$ - matrix

$$
T_{(i)(j)}\left(\boldsymbol{k}, \boldsymbol{k}^{\prime}\right)=\sum_{(\ell)} \varrho^{(\ell)}\left(\boldsymbol{k}-\boldsymbol{k}^{\prime}\right) \tau_{(i)(j)}^{(\ell)}\left(\boldsymbol{k}, \boldsymbol{k}^{\prime}\right) .
$$

- An empty square stands for a $\sigma$-matrix:

$$
\sigma_{(i)(j)}^{(\ell)}=2 g \delta_{(i)(j)} \delta_{(i)(\ell)} \bar{\mu}_{(\ell)} .
$$

- The $S$-matrix is depicted as an empty circle and is given by

$$
S_{(i)(j)}\left(\boldsymbol{k}, \boldsymbol{k}^{\prime}\right)=\sum_{(\ell)} \varrho^{(\ell)}\left(\boldsymbol{k}-\boldsymbol{k}^{\prime}\right) \sigma_{(i)(j)}^{(\ell)} .
$$

- The propagator of uncoupled magnetic moments, represented by a solid line, is given as

$$
\Gamma_{(i)(j)}(z)=z^{-1} \delta_{(i)(j)} .
$$

- A cumulant of order $n$ is represented by a crossed circle, where the order is given by the number of dashed lines ending at it.

Furthermore, two rules for the interpretation of the diagrams need to be followed:

(a) The elements brought together in a diagram undergo a matrix multiplication in the $(i)(j)$-space. The corresponding matrix indices are written as subscripts in the definitions above.

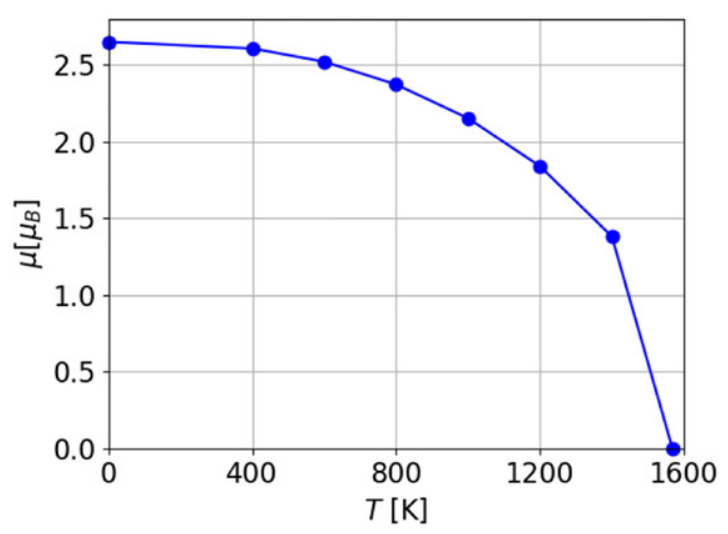

Figure 3. Calculated temperature dependence of the magnetic moments' $z$ component. The critical temperature lies at $T_{\mathrm{C}}=1569 \mathrm{~K}$.

(b) Every internal free propagator is assigned a momentum which is integrated over:

$$
\frac{1}{\Omega_{\mathrm{BZ}}} \int_{\Omega_{\mathrm{BZ}}} \mathrm{d}^{3} k_{1}
$$

Averaging over all possible configurations needs to be done carefully to obtain correct magnetic properties of the material. The average of the Fourier transformed second term in series 3 is depicted in figure 2(b). Starting from the fourth order diagrams, diagrams with crossed dashed lines will appear. These diagrams correspond to correlations between the occupation of different sites. In our approach, these diagrams are neglected. Since the averaged diagrams consist of two different types of vertices (filled and empty squares), the averaged susceptibility $\mathcal{X}$ can be written as a product of two quantities as shown in figure 2(c). These two quantities are defined in figures 2(d)-(f). It can be shown that all non-crossed diagrams can be constructed with these definitions. The self consistency of the method is evident from the fact that the self energy (figure 2(e)) depends on the effective medium propagator which in turn depends on the self energy. Further details of the theory are given in references $[14,19,30]$.

\section{Results}

Magnetic moments $\mu_{i}^{\alpha}$ and exchange parameters $J_{i j}$ of iron-cobalt alloys at various concentrations were evaluated using a first-principles Green-function method within a generalized gradient approximation of density functional theory [31]. The method is designed for bulk materials, surfaces, interfaces and real space clusters [32-34]. The resulting magnetic moments read $\mu_{\mathrm{Fe}}=2.65 \mu_{\mathrm{B}}$ and $\mu_{\mathrm{Co}}=1.83 \mu_{\mathrm{B}}$. The average magnetic moment per atom is given by the arithmetic mean of the constituents magnetic moments (for 50:50 alloys) and reads $\mu=2.24 \mu_{\mathrm{B}}$, which is in good agreement with experimental results [35]. The impact of disorder on the electronic structure was taken into account within the electronic CPA [36] implemented within multiple scattering theory [37]. Exchange interaction was estimated using the magnetic force theorem [26] formulated for substitutional alloys within the 

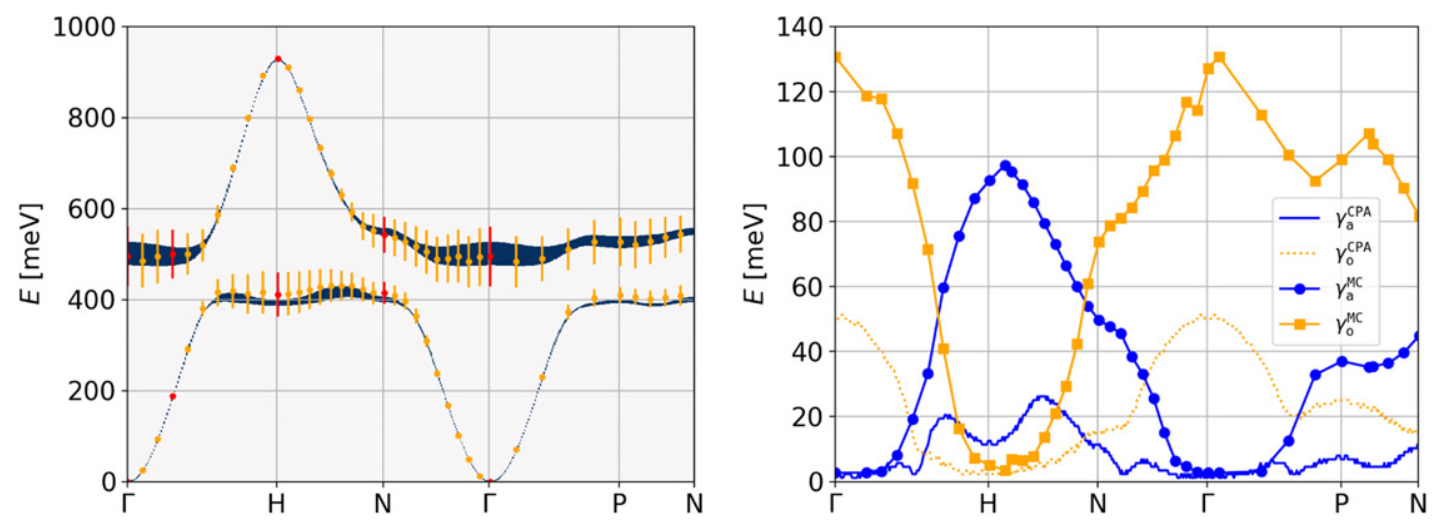

Figure 4. Comparison of the magnonic spectra (left) obtained through the CPA (blue lines) and MC (orange and red dots) calculations. The errorbars for the MC-results represent the width of the peaks as does the line width for the CPA results. In the right figure the absolute values of the peak widths are shown.

CPA approach [38]. Although both iron and cobalt are known for long ranged interaction between the magnetic moments, the results presented in this section use only 12 shells of neighbors because of computational limits. To ensure the convergence of spin waves with the number of neighbor shells and supercell size, several calculations were performed for 30 neighbor shells showing essentially the same results as with 12 shells. We consider only the disordered alloy $\mathrm{Fe}_{0.5} \mathrm{Co}_{0.5}$.

For better comparability the bcc structure was taken in all calculations. Furthermore, the interaction parameters $J_{i j}$ are held constant (at their value at $T=0 \mathrm{~K}$ ). Both $\mathrm{MC}$ and CPA calculations are done at complex energies with a small imaginary part $\epsilon=10^{-4}$ Ry.

The resulting temperature dependence of the magnetic moments is shown in figure 3. Although the RPA is known to underestimate the Curie temperature [39], we obtain a Curie temperature above the experimental values of $T_{\mathrm{C}} \approx 1250 \mathrm{~K}$ $[12,13]$. This behavior can be partly explained by the fact that the real FeCo system will perform a structural phase transition, while in the calculations the structure (bcc) was fixed.

\subsection{Eigenmodes at $T=0$}

The magnonic spectra (cf figure 4) obtained using both methods are basically identical. In the left plot of figure 4, the density plot in the background represents the imaginary part of the CPA susceptibility while the dots stand for the position of the susceptibility peaks within the MC. The errorbars at the points represent the full width at half maximum of the peaks. The same holds for the width of the line and the CPA results. Due to the finite size of the supercell, the MC can only give meaningful results for a discrete set of points within the Brillouin zone (BZ). The spectra are in good agreement with other results for ordered FeCo systems $[40,41]$ (note that the spectra in these references are calculated for a $\mathrm{CsCl}$ structure).

We obtain a spin wave stiffness of $C \approx 675 \mathrm{meV} \AA$ at $T=0 \mathrm{~K}$. Experimental values range from $C \approx 500 \mathrm{meV} \AA$ [42] (at $T=0 \mathrm{~K}$ ) to $C \approx 800 \mathrm{meV} \AA$ [43] (thin film at room temperature). We note that the long range interaction between magnetic moments which was neglected in this work due to computational limits may influence the magnonic spectrum, especially close to the $\Gamma$ point and therefore also affect the spin stiffness.

It can clearly be seen that the CPA systematically underestimates the width of the peaks. In the right plot of figure 4, we show the full width at half maximum $\gamma$ for the acoustic and optic modes within the CPA and the MC calculations. Although the absolute values of the damping is different, the general trend of $\gamma$ through the considered paths in the BZ is very similar. The fact that the $\mathrm{MC}$ calculations give much larger widths indicates that non-local effects play an essential role in the damping of magnons. Recent studies [44-46] reveal, that non-local effects also play an important role in the Ising model. Although the Ising model only captures nearest neighbor spin interaction, in 3D a term with an effective long range interaction appears in the partition function [45]. Although the results of these studies cannot be directly applied to our model, they represent a further hint at the presence and influence of non-local effects.

The CPA predicts [14] that at the $50 \%$ concentration of cobalt the bandgap is still present. However, the more realistic estimation of the magnon damping using the MC method tends to suggest that the significant widening of the magnon modes might lead to effective closing of the gap.

The red dots in figure 4 mark the $k$-points for which the spatial shapes of the magnon modes will be analyzed in more detail now. In figure 5 the eigenvalues and eigenvectors of the loss matrix are shown at different symmetry points in the BZ and $T=0$. The maximal eigenvalue at the $\Gamma$ point for $T=0$ (figure 5, top left) exhibits the Goldstone mode at zero energy, i.e. the mode where the moments of both constituents are tilted by the same amount.

As already mentioned, we explain the larger width of the MC peaks with the influence of correlation effects between different sites, which the CPA cannot account for. The appearance of smaller peaks at regions where the CPA susceptibility is effectively zero is a further effect caused by non local effects. An interesting fact is that at the $\mathrm{N}$ point (cf bottom right plot in figure 5) essentially only one of the constituents precesses for both the acoustic and the optic mode. Unfortunately, the direct measurement of the shape very challenging but recent studies suggest that it could be realized on the surface of a 2D 

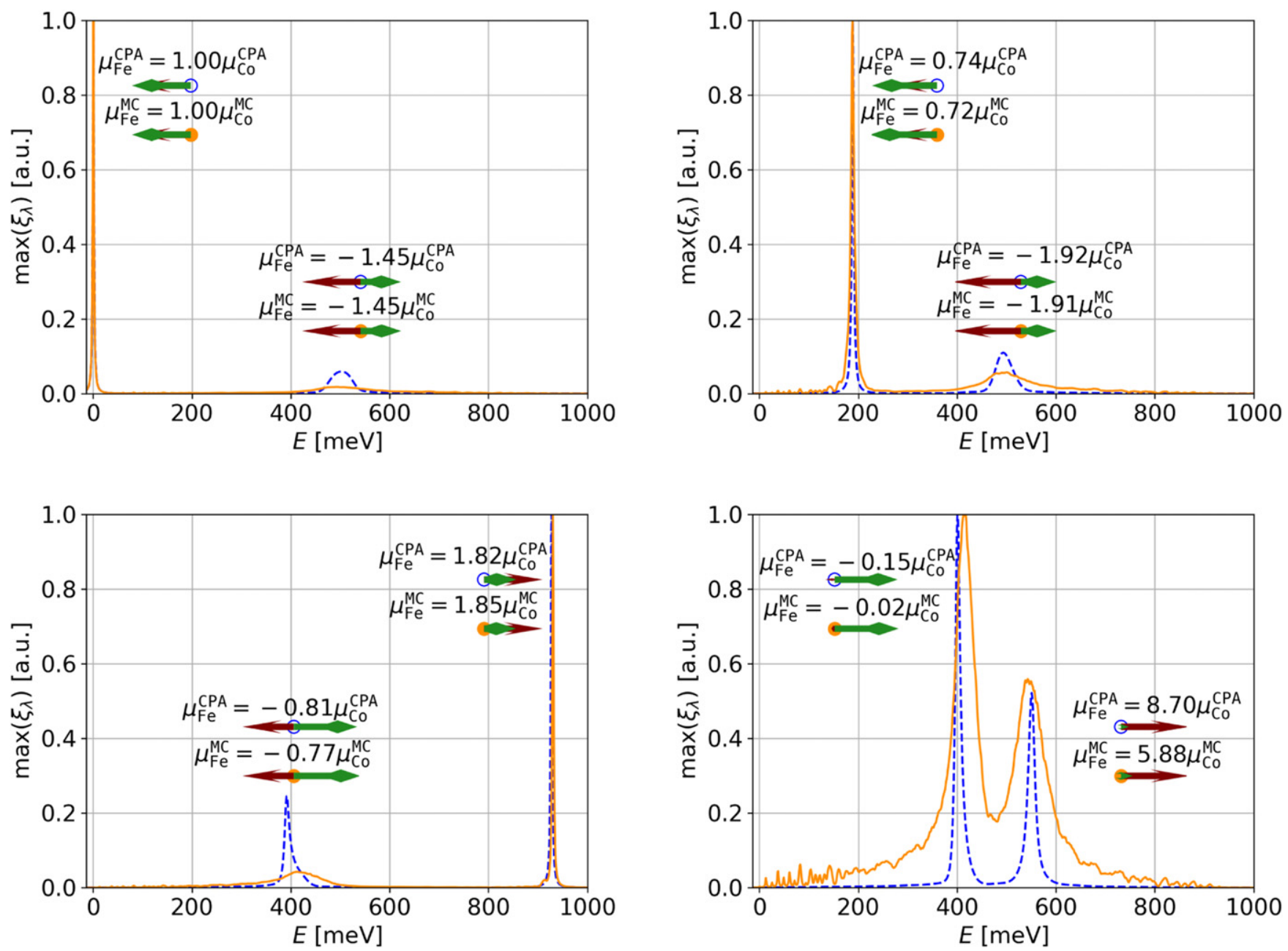

Figure 5. Maximal eigenvalue of the loss matrix at $\Gamma$ (top left), $\frac{\Gamma \mathrm{H}}{3}$ (top right), $\mathrm{H}$ (bottom left) and $\mathrm{N}$ (bottom right). All results are calculated at $T=0 \mathrm{~K}$ within the MC (orange solid line) and CPA (blue dashed line). The spatial shape of the modes is drawn near the peaks. Starting from a blue empty circle (CPA) or an orange filled circle (MC), the magnetic moments of iron are drawn as brown pointy vectors while the moments of cobalt are visualized as green edgeless vectors.

magnet using atomic resolution inelastic scanning tunneling microscopy [47].

Generally, we come to the conclusion that the CPA is able to precisely give the magnetic properties of this iron cobalt alloy apart from a systematic underestimation of the magnon damping.

\subsection{Spatial form of the eigenmodes}

Through the diagonalization of the susceptibility in real space (equation (3)), the spatial eigenvectors at a certain energy can be directly found through the loss matrix (equation (6)). We did so calculating the real space susceptibility of one specific random configuration in a $30 \times 30 \times 10$ atom supercell. The eigenmode corresponding to the highest eigenvalue of the loss matrix at $E \approx 200 \mathrm{meV}$ is depicted for this specific random configuration in figure 6 . This figure shows one plane of the supercell corresponding to $\boldsymbol{R}=\lambda_{1} \mathbf{a}_{1}+\lambda_{2} \mathbf{a}_{2}+5 \mathbf{a}_{3}$ with the primitive lattice vectors $\boldsymbol{a}_{i}, \lambda_{1}, \lambda_{2} \in \mathbb{N}$ and $\lambda_{1}, \lambda_{2} \leqslant 30$.

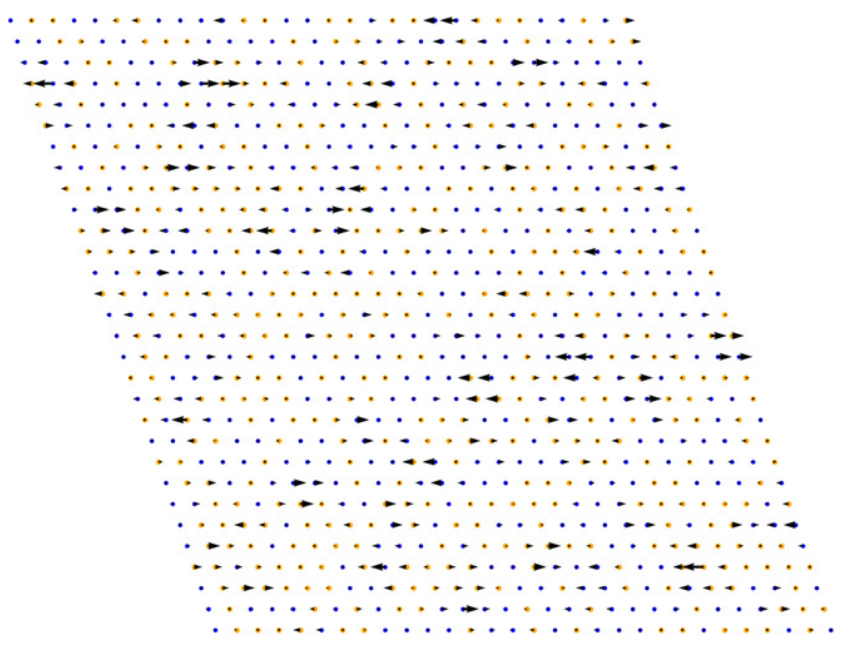

Figure 6. Real space mode of the susceptibility (corresponding to the highest eigenvalue) at $E=200 \mathrm{meV}$. The blue/orange dots represent iron/cobalt atoms. 

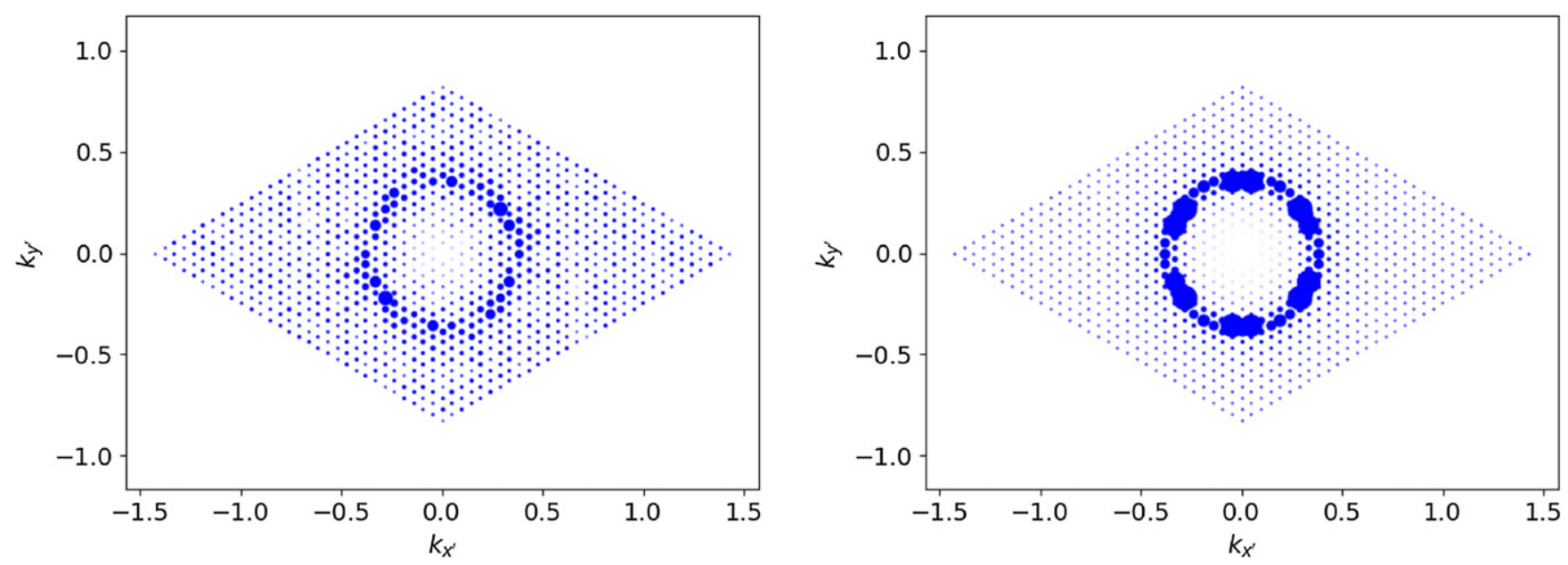

Figure 7. Fourier components of the eigenmode corresponding to the highest eigenvalue $\xi_{1}$ (left) and the loss matrix projected on plain wave states (right) for different reciprocal lattice vectors. The plane shown in the figure correspond to the space spanned by the reciprocal lattice vectors $b_{1}=\left(0, \frac{2 \pi}{a}, \frac{2 \pi}{a}\right)$ and $b_{2}=\left(\frac{2 \pi}{a}, 0, \frac{2 \pi}{a}\right)$.
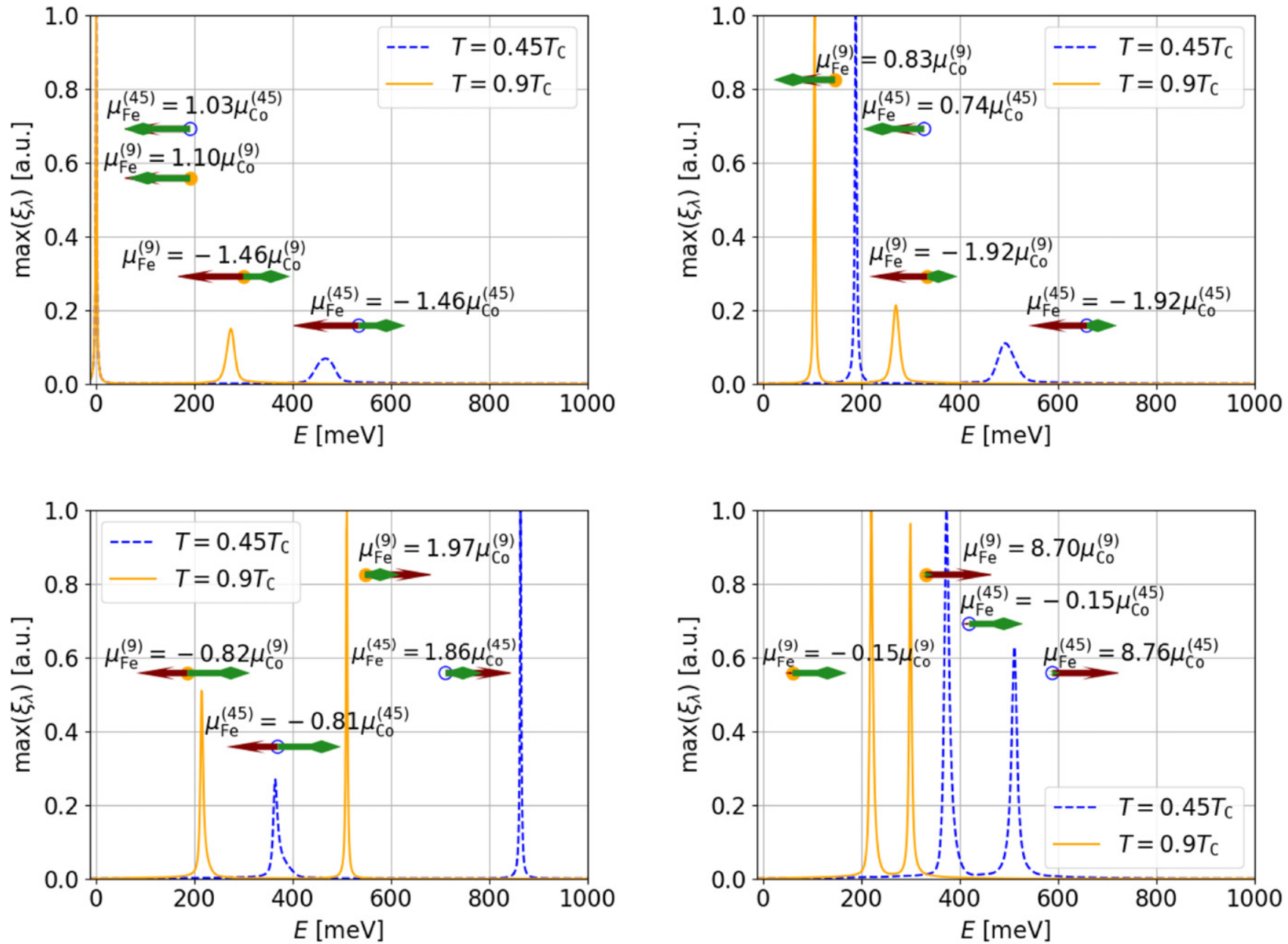

Figure 8. Maximal eigenvalue of the loss matrix at $\Gamma$ (top left), $\frac{\Gamma \mathrm{H}}{3}$ (top right), $\mathrm{H}$ (bottom left) and $\mathrm{N}$ (bottom right). All results are calculated at $T=0.45 T_{\mathrm{C}}$ and $T=0.9 T_{\mathrm{C}}$ within the CPA. The spatial shape of the modes is drawn near the peaks. Starting from a blue empty circle $\left(T=0.45 T_{\mathrm{C}}\right)$ or an orange filled circle $\left(T=0.9 T_{\mathrm{C}}\right)$, the magnetic moments of iron are drawn as brown pointy vectors while the moments of cobalt are visualized as green edgeless vectors. 
The orange dots correspond to Co atoms while the blue dots represent $\mathrm{Fe}$ atoms. It is assumed that in the magnetic ground state all magnetic moments are oriented orthogonal to the plane depicted. The in-plane components of the magnetic moments $\tilde{\mu}_{i}$ of this particular mode are represented by the arrows. Interestingly, we observe that the mode shown in figure 6 features multiple small clusters of precessing moments.

We further analyze the eigenmode by performing a Fourier analysis of the $\tilde{\mu}_{i}$

$$
\tilde{\mu}_{\boldsymbol{k}}=\frac{1}{N} \sum_{i=1}^{N} \mathrm{e}^{\mathrm{i} \boldsymbol{k} \cdot \boldsymbol{R}_{i}} \tilde{\mu}_{i}
$$

with the positions of the atoms $\boldsymbol{R}_{i}$ and the number of atoms $N=9000$ in our case. The result is depicted in figure 7 for a plane in the BZ. The dominant contributions arise from wavevectors lying on an circle. In an ordered system, the circle would be the cross section of the constant energy surface in reciprocal space. Due to the disorder, Bloch waves cease to be eigenstates of the system (the corresponding peaks acquire finite widths) and the eigenstates pick up Fourier components outside of a single energy surface. Finally, we further analyze the loss matrix by writing its spectral representation

$$
\mathcal{L}_{i j}(E)=\sum_{\lambda} \xi_{\lambda}\left|\tilde{\mu}_{i}^{\lambda}\right\rangle\left\langle\tilde{\mu}_{j}^{\lambda}\right|
$$

with the eigenvalues $\xi_{\lambda}$ and eigenvectors $\left|\tilde{\mu}_{i}^{\lambda}\right\rangle$. The projection of the loss matrix to plain wave states is then given by

$$
\langle\boldsymbol{k}|\mathcal{L}(E)| \boldsymbol{k}\rangle=\sum_{\lambda} \xi_{\lambda}(E)\left|\tilde{\mu}_{\boldsymbol{k}}^{\lambda}\right|^{2}
$$

which represents a weighted sum of Fourier components. The plain wave projection for all eigenvalues larger than $\frac{\xi_{1}}{100}$, with the highest eigenvalue $\xi_{1}$, is given in figure 7 . Considering all the modes with significant eigenvalues at this energy recovers the picture of the constant energy surface similar to the one of an ordered system. While a single eigenmode is clearly different from a Bloch wave, the dynamics of the entire system resembles the one of the ordered system. In a sense, the Fourier transformation recovers the self-averaging property of the spin dynamics in disordered magnets.

\subsection{Eigenmodes at finite temperatures}

Next, we investigate the change of the eigenmodes with temperature. It turned out that our realization of the RPA in combination with the MC calculations is computationally too expensive for the system size we are considering here. Therefore, we restrict this discussion to the CPA + RPA results and stress again the agreement of CPA and MC shown in the previous section especially when it comes to the spatial form of the modes. We recall that the widely used RPA [48-50] cannot account for the temperature broadening of the magnon modes [14] such that we must restrict ourselves to the analysis of the impact of the temperature on the shapes of magnetic modes, $\mathrm{cf}$ figure 8. Our calculations suggest that the spatial shape of the modes is independent of temperature.

\section{Summary}

We provided a thorough analysis of the magnonic modes in a disordered iron cobalt alloy using two complementary numerical schemes. The MC and the CPA give basically the same magnonic properties apart from the disorder induced width of the peaks in the magnonic spectrum. We explain this discrepancy with non-local effects which the single site CPA cannot account for. Interestingly, we found that the acoustic and optic mode at the $N$ point features the precession of only one of the constituents' magnetic moments. The eigenspectrum analysis of the loss matrix in real space reveals that the eigenmodes involve many small clusters of precessing magnetic moments. We are convinced that such real space effects can be used to effectively excite only defining parts of the magnonic crystal and thus gain additional precise control over the spin dynamics of the system. Finally, it turns out that the spatial shape of the modes is basically invariant with respect to temperature.

\section{Data availability statement}

All data that support the findings of this study are included within the article (and any supplementary files).

\section{ORCID iDs}

S Paischer (D) https://orcid.org/0000-0002-1469-3755

\section{References}

[1] Chumak A V, Vasyuchka V I, Serga A A and Hillebrands B 2015 Nat. Phys. 11 453-61

[2] Bloch F 1930 Z. Phys. 61 206-19

[3] Chumak A V, Serga A A and Hillebrands B 2017 J. Phys. D: Appl. Phys. 50244001

[4] Nikitov S A, Tailhades P and Tsai C S 2001 J. Magn. Magn. Mater. 236 320-30

[5] Krawczyk M and Grundler D 2014 J. Phys.: Condens. Matter. 26123202

[6] Lenk B, Ulrichs H, Garbs F and Münzenberg M 2011 Phys. Rep. 507 107-36

[7] Sadovnikov A V, Beginin E N, Odincov S A, Sheshukova S E, Sharaevskii Y P, Stognij A I and Nikitov S A 2016 Appl. Phys. Lett. 108172411

[8] Sadovnikov A V, Gubanov V A, Sheshukova S E, Sharaevskii Y P and Nikitov S A 2018 Phys. Rev. Appl. 9

[9] Sheshukova S E, Morozova M A, Beginin E N, Sharaevskii Y P and Nikitov S A 2013 Phys. Wave Phenom. 21 304-9

[10] Zakeri K 2018 Physica C 549 164-70

[11] Buczek P, Ernst A, Bruno P and Sandratskii L M 2009 Phys. Rev. Lett. 102247206

[12] Normanton A S, Bloomfield P E, Sale F R and Argent B B 1975 Metal Sci. $9510-7$

[13] Nishizawa T and Ishida K 1984 Bull. Alloy Phase Diagrams 5 250-9

[14] Paischer S, Buczek P A, Buczek N, Eilmsteiner D and Ernst A 2020 Spin waves in disordered $\mathrm{FeCo}$ magnonic crystal at finite temperatures Phys. Rev. B (arXiv:2009.04712)

[15] Buczek P, Ernst A and Sandratskii L M 2011 Phys. Rev. Lett. 106157204 
[16] Buczek P, Ernst A and Sandratskii L M 2011 Phys. Rev. B 84 174418

[17] Zakeri K, Zhang Y, Chuang T H and Kirschner J 2012 Phys. Rev. Lett. 108197205

[18] Qin H J, Zakeri K, Ernst A, Sandratskii L M, Buczek P, Marmodoro A, Chuang T H, Zhang Y and Kirschner J 2015 Nat. Commun. 66126

[19] Buczek P, Sandratskii L M, Buczek N, Thomas S, Vignale G and Ernst A 2016 Phys. Rev. B 94054407

[20] Edström A et al 2015 Phys. Rev. B 92174413

[21] Rusz J, Bergqvist L, Kudrnovský J and Turek I 2006 Phys. Rev. B 73214412

[22] Callen H B 1963 Phys. Rev. 130 890-8

[23] Şaşığlu E, Friedrich C and Blügel S 2013 Phys. Rev. B 87 020410

[24] Staunton J B, Poulter J, Ginatempo B, Bruno E and Johnson D D 1999 Phys. Rev. Lett. 82 3340-3

[25] Staunton J B, Poulter J, Ginatempo B, Bruno E and Johnson D D 2000 Phys. Rev. B 62 1075-82

[26] Liechtenstein A I, Katsnelson M I, Antropov V P and Gubanov V A 1987 J. Magn. Magn. Mater. 67 65-74

[27] Nolting W and Ramakanth A 2009 Quantum Theory of Magnetism (Berlin: Springer)

[28] Kubo R 1966 Rep. Prog. Phys. 29 255-84

[29] Yonezawa F 1968 Prog. Theor. Phys. 40 734-57

[30] Buczek P et al 2018 J. Phys.: Condens. Matter. 30423001

[31] Perdew J P, Burke K and Ernzerhof M 1996 Phys. Rev. Lett. 77 $3865-8$
[32] Lüders M, Ernst A, Temmerman W M, Szotek Z and Durham P J 2001 J. Phys.: Condens. Matter. 13 8587-606

[33] Geilhufe M, Achilles S, Köbis M A, Arnold M, Mertig I, Hergert W and Ernst A 2015 J. Phys.: Condens. Matter. 27 435202

[34] Hoffmann M, Ernst A, Hergert W, Antonov V N, Adeagbo W A, Geilhufe R M and Ben Hamed H 2020 Phys. Status Solidi B 2571900671

[35] Goldman J E and Smoluchowski R 1949 Phys. Rev. 75 310-1

[36] Soven P 1967 Phys. Rev. 156 809-13

[37] Gyorffy B L 1972 Phys. Rev. B 5 2382-4

[38] Turek I, Kudrnovský J, Drchal V and Bruno P 2006 Phil. Mag. 86 1713-52

[39] Rusz J, Turek I and Diviš M 2005 Phys. Rev. B 71174408

[40] Okumura H, Sato K and Kotani T 2019 Phys. Rev. B 100054419

[41] Grotheer O, Ederer C and Fähnle M 2001 Phys. Rev. B 63 100401

[42] Lowde R D, Shimizu M, Stringfellow M W and Torrie B H 1965 Phys. Rev. Lett. 14 698-700

[43] Liu X, Sooryakumar R, Gutierrez C J and Prinz G A 1994 J. Appl. Phys. 75 7021-3

[44] Zhang Z-D 2007 Phil. Mag. 87 5309-419

[45] Zhang Z D 2017 J. Phys.: Conf. Ser. 827012001

[46] Zhang Z 2020 J. Mater. Sci. Technol. 44 116-20

[47] Hirjibehedin C F 2006 Science 312 1021-4

[48] Tang G X and Nolting W 2006 Phys. Rev. B 73024415

[49] Bouzerar G and Bruno P 2002 Phys. Rev. B 66014410

[50] Matsubara T 1973 Prog. Theor. Phys. Suppl. 53 202-21 